\title{
1 Response of whitefly to the wild tomato Solanum
}

\section{2 habrochaites}

3 Fengqi Li ${ }^{1, *}$, Youssef Dewer ${ }^{2}$, Jiahui Tian' ${ }^{1}, \mathrm{Du} \mathrm{Li}^{1}$, Cheng $\mathrm{Qu}^{1}$, Zhen

$4 \quad$ Yang $^{3} \&$ Chen Luo

5

6

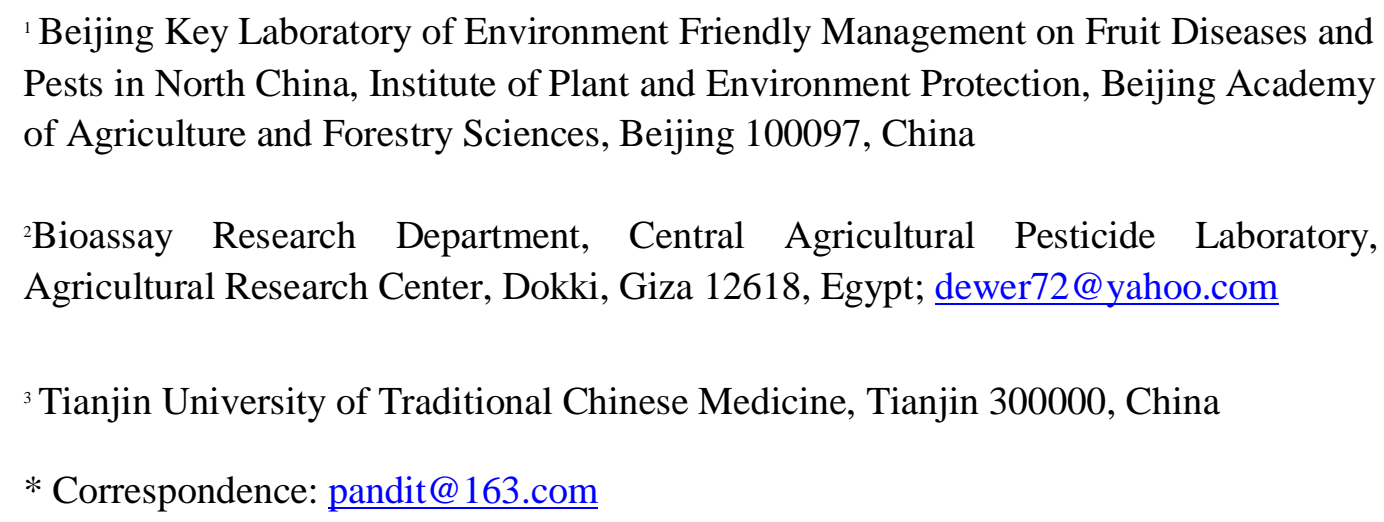

\section{Abstract}

The whitefly Bemisia tabaci (Gennadius) causes severe damage to cultivated tomato in many regions of the world through direct feeding and indirectly through transmission of plant viruses. Field observations show that B. tabaci is rarely infested the non-host plants such as the wild tomato Solanum habrochaites; however, the molecular mechanism involved in the recognition of wild plant odors is still unclear. In this study, we assessed the effects of S. habrochaites on the survival, fecundity, and egg hatchability of the Mediterranean (MED) species of B. tabaci. Expression and splicing of stress-response genes in whitefly exposed to S. habrochaites was analyzed using RNA-sequencing data and alternative splicing analysis. These results indicated that the $\mathrm{S}$. habrochaites treatment can induce the expression of environmental stress genes in B. tabaci. This study may help us to a better understanding of the molecular 
27 mechanisms involved in the olfactory recognition of non-host volatiles particularly

wild tomato relative. Furthermore, the findings of this study may provide excellent chances of finding a suitable antagonist of eco-friendly properties which can block the perception of chemosensory signals. Thereby, the feeding behavior and food preferences of B. tabaci can be manipulated and thus insect populations can eventually be controlled.

Keywords: whitefly, Solanum habrochaites, transcriptome

\section{Introduction}

The Whitefly Bemisia tabaci (Gennadius) is one of the most important pests that causes economic damage to crop plants (De et al., 1997). B. tabaci has a high oviposition rate and rapid population growth (Erdogan et al., 2008). The Middle East-Asia Minor1 (MEAM1) and Mediterranean (MED) subspecies are the most invasive B. tabaci whiteflies worldwide (Chu et al., 2006). In China, the MED cryptic species has now been replaced by MEAM1 in many regions (Hu et al., 2011).

B. tabaci is one of the main tomato pests that reduces tomato growth through direct feeding and indirectly through transmission tomato yellow leaf curl virus (TYLCV) (Su et al., 2016). B. tabaci is the only vector of TYLCV, so whitefly control is one of the key measures used to manage TYLCV (Wei et al., 2017). Chemical insecticides remain the main tools in the management of whitefly, but the rapid development of resistance to several chemical classes of insecticides has made it so difficult to control (Elbert et al., 2000). Therefore, there are urgent demands for safe alternative strategies in modern pest management, i.e. for controlling whitefly populations. One of these 
novel approaches is based on the fact that chemical communication via the olfactory system drives essential behaviors of insects (Ingham et al., 2020). Indeed, detecting appropriate chemical signals is essential for insects to find their mating partners, oviposition sites and food sources (Pelosi et al., 2017; Leal and Walter, 2013). Accordingly, the olfactory system of many insects has evolved to chemo-detectors of high sensitivity and accuracy, which allow the insect to detect plant volatile odors at extremely low concentrations and to discriminate a large variety of odor cues (Suh et al., 2014) . Thus, understanding of the mechanisms and signals that are involved in non-host selection behavior of $B$. tabaci may lead to strategies that exploit the odorous alert signals to enhance crop resistance.

B. tabaci is attracted to cultivated tomato, but it is repelled by the wild tomato relative $S$. habrochaites. The repellent behavior response of $B$. tabaci exposed to $S$. habroachaites as non-host plant was found to be associated with the emission of 7-epizingiberene and R-curcumene compounds (Bleeker et al., 2009). This two terpene volatiles have shown strong repellency against B. tabaci and induced $S$. habrochaites to develop enhanced resistance to insect infestation (Freitas et al., 2002; Muigai et al., 2002). On the other hand, B. tabaci did not elicit behavioral responses to the two corresponding chiral isomers: $\alpha$-zingiberene and S-curcumene extracted from ginger (Bleeker et al., 2011). Transformation of cultivated tomato with the gene encoding S. habrochaites 7-epizingiberene synthase has become strongly repellent to B. tabaci that reduced whitefly fecundity up to $87 \%$ (Bleeker et al., 2012). These studies showed that $S$. habrochaites can exert strong repellent and toxic stresses 
aganist B. tabaci, but it is unclear how it exerts its effects on the physiological and molecular levels.

$$
\text { In order to achieve the goal, it was necessary to study the expression-related genes }
$$
that may be affected in the whitefly B. tabaci after exposure to the wild tomato relative $S$. habrochaites.

\section{Materials and methods}

\subsection{Insects rearing and plant materials}

The MED cryptic species of whitefly was reared on tomato plants (Xianke 8) under greenhouse conditions of $25 \pm 2{ }^{\circ} \mathrm{C}, 65 \pm 5 \%$ relative humidity $(\mathrm{RH})$, and a 16:8 $\mathrm{h}$ (L:D) photoperiod. The tomato plant was placed into cage measuring $(60 \mathrm{~cm} \times 60 \mathrm{~cm}$ $\times 60 \mathrm{~cm})$. The source of MED was collected from a greenhouse located in Beijing, China and identified as MED by mitochondrial cytochrome oxidase I (mtCOI) gene (Brown et al., 2005). One pair of newly emerged adults was used for colony initiation, and the MED source after six generations was used for subsequent experiments.

\subsection{Identification of wild tomato volatiles}

S. habrochaites were placed into the cages measuring $(60 \mathrm{~cm} \times 60 \mathrm{~cm} \times 60 \mathrm{~cm})$ and grown under greenhouse conditions of $25 \pm 2^{\circ} \mathrm{C}, 65 \pm 5 \% \mathrm{RH}$, and a 16:8 h (L:D) photoperiod. Six-week-old plants were used for volatile analysis. We placed leaves of a healthy tomato plant into a glass bottle $(45 \mathrm{~mL}$, cleman) and an SPME fiber 50/30- $\mu \mathrm{m}$ divinylbenzene/carboxen/polydimethylsiloxane (Supelco; Sigma-Aldrich, http://www.sigmaaldrich.com) was used for analyte extraction. GC-MS analysis was performed on an Agilent 7890 (Agilent Technologies, Tustin, CA, USA) gas 
chromatography and Agilent 5975 mass selective detector.. Samples were separated using both HP-5 and DB-WAX (both $30 \mathrm{~m} \times 0.25 \mathrm{~mm}$ i.d., $0.25 \mathrm{~mm}$ film thickness, Agilent Technologies, Tustin, CA, USA). Helium was used as carrier gas at a flow rate of $1.7 \mathrm{~mL} / \mathrm{min}$, and the GC inlet was set in the split-less mode. The injector temperature was $220{ }^{\circ} \mathrm{C}$. The optimization of method parameters of SPME and GC-MS were performed following to the method previously described ( $\mathrm{Li}$ et al., 2018). R-curcumene has been identified using GC-MS techniques by comparing its retention time and mass spectrum with the same parameters from a reference compound. As the 7-epizingiberene is thermally unstable (Bleeker et al., 2011), therefore, the R-curcumene was used for conducting the behavioral and olfactory studies. The synthesis of R-curcumene and S-curcumene was performed according to the method previously reportedS (Song et al., 2012). The synthesized compounds were confirmed by using various spectral technique like mass spectrometry and NMR detection. The $\alpha$-zingiberene was purchased from Sigma-Aldrich (St. Louis, MO, USA).

\subsection{RNA sequencing and bioinformatics analysis}

To evaluate the effect of $S$. habrochaites on whiteflies at the molecular level, RNA-sequencing was performed using MED females and males, which have been fed on tomato Xianke 8 and the wild tomato S. habrochaites LA2175 for 6 and $24 \mathrm{~h}$, respectively. Sequencing libraries were constructed using strand-specific libraries. FastQC (Andrews et al., 2010) was used to assess the quality of the sequencing data while Fastp (Chen et al., 2018) was employed to remove adapters, contamination, and 
115 duplicate sequences. The sequenced clean data were mapped to the MED whitefly

116 genome v1.0 (Xie et al., 2017) using HISAT2 (Kim et al., 2019)

117 ftp://www.whiteflygenomics.org/pub/whitefly/MED/v1.0. FeatureCounts (Liao et al.,

118 2014) was used to calculate the expression of each gene, edgeR (Roboison et al., 2010)

119 was used to analyze the difference in gene expression (we used Log2 Fold Change>1,

120 Adjusted P-value $=0.05$ as the threshold), rMATS (Shen et al., 2014) was used to

121 analyze the alternative splicing, and Sashimi plot was used to draw the alternative

122 splicing of genes. Finally, WGCNA (Langfelder and Horvath, 2008) was used to

123 calculate the correlation between the gene expression level (TPM values) and the

124 mortality phenotypes of whitefly.

\section{3. Results and discussion}

126

127

128

129

130

132

133

134

135

136

3.1 7-epizingiberene and R-curcumene are the main volatile components of $S$.

\section{habrochaites}

We synthesized a high-purity R-curcumene and confirmed its identity by mass spectrometry and NMR (Supplementary Data 1). We found that 7-epizingiberene and R-curcumene are the main volatile compounds of S. habrochaites using SPME and GC-MS detection. GC-MS analysis revealed that these two volatile compounds were not detected in the volatile blends of common tomato plants (Fig. 1). It was also found that the peak areas ratio of 7-epizingiberene and R-curcumene in LA2175 was 2.37and 4.27-fold that of LA2329, respectively (Fig. 1). The data showed that LA2175 leaves had a relatively higher content of terpene volatiles than LA2329. This is consistent with the biological analysis showing that LA2175 was more resistance to 
137 whiteflies. Therefore, we chose and employed LA2175 for subsequent whitefly

138 high-throughput sequencing studies.

$139 \quad 3.2$ Expression of whitefly-related genes after stress exposure of $S$. habrochaites

140 A total of 24 samples of male and female whitefly adults (including two time points

141 and four treatments) were used for transcriptome sequencing (Fig. 2). About 21-24

142 million reads with an average length of $151 \mathrm{bp}$ were aligned to the MED whitefly

143 reference genome by HISAT2 (Table 1). About $78 \%$ of the reads mapped to unique

144 loci (Table 1). After gene expression value analysis by featurecount and edgeR, we

145 found four significantly upregulated genes in wild tomato 6-h-treated female adults

146 (WF6) and wild tomato 24-h-treated female adults (WF24) (Fig. 2; Supplementary

147 Data 2). They were BTA016109 (Multicopper oxidase), BTA027707

148 (Sulfotransferase), BTA001651 (OHCU decarboxylase), and BTA011604 (Heat shock

149 protein). Wild tomato 6-h-treated male adults (WM6) and wild tomato 24-h-treated

150 female adults (WM24) also included four genes that are both significantly upregulated

151 (Fig. 2; Supplementary Data 2). They were BTA003886 (Heat shock protein),

152 BTA016667 (Unknown), BTA005900 (serine threonine-protein kinase SBK1-like),

153 and BTA011604 (Heat shock protein). One gene (BTA011604, Heat shock protein)

154 was significantly upregulated together in these four treatments. There were two genes

155 that were significantly downregulated in WF6 and WF24, BTA009028 (Unknown)

156 and BTA013802 (Farnesyl-diphosphate farnesyltransferase). Eighteen genes were

157 significantly downregulated in WM6 and WM24 (Fig. 2; Supplementary Data 2).

158 Among these, BTA022129 is solute carrier family 3, member 1, and the other genes 
159

160

161

162

163

164

165

166

167

168

169

170

171

172

173

174

175

176

177

178

179

180

had unknown functions. These results indicated that the $S$. habrochaites treatment can induce the expression of environmental stress genes such as heat shock protein in whiteflies.

Alternative splicing is often related to the response of the organism to a stressful environment (Sultan et al., 2008; Ling et al., 2015; Wang et al., 2008). We determined if whiteflies were stressed by exposure to $S$. habrochaites and whether genes were able to respond to the stress through alternative splicing. We performed alternative splicing analysis on differently treated samples (comparison of male and female samples treated with $S$. habrochaites and then treated with common tomato) through rmats. There were five types of alternative splicing, including skipped exon (SE), mutually exclusive exon (MXE), alternative to 5'splice site (A5SS), alternative to $3^{\prime}$ splice site (A3SS), and retained intron (RI). Among these, only SE and MXE had significant differences between the two treatments (FDR <0.05), and the SE type accounted for the main proportion (Table 2; Supplementary Data 3). GO enrichment analysis showed that genes with alternative splicing of the SE type were significantly enriched in three GO items including O-acetyltransferase activity, glycerol ether metabolic process, and ether metabolic process (Supplementary Data 4). The MXE-type alternative splicing genes were not significantly enriched.

\section{Data availability conflict of interest}

RNA-seq data have been deposited and are available in NCBI (BioProject ID is PRJNA622388).

Conflict of interest: The authors declare no conflict of interest. 


\section{References}

Andrews, S., FastQC: a quality control tool for high throughput sequence data. Babraham Bioinformatics, Babraham Institute, Cambridge, United Kingdom, 2010.

Bleeker, P. M., et al., 2009. The role of specific tomato volatiles in tomato-whitefly interaction. Plant Physiology. 151, 925-935.

Bleeker, P. M., et al., 2011. Tomato-produced 7-epizingiberene and R-curcumene act as repellents to whiteflies. Phytochemistry. 72, 68-73.

Bleeker, P. M., et al., 2012. Improved herbivore resistance in cultivated tomato with the sesquiterpene biosynthetic pathway from a wild relative. Proceedings of the National Academy of Sciences. 109, 20124-20129.

Chen, S., et al., 2018. fastp: an ultra-fast all-in-one FASTQ preprocessor. Bioinformatics. 34, i884-i890.

Chu, D., et al., 2006. The introduction of the exotic Q biotype of Bemisia tabaci from the Mediterranean region into China on ornamental crops. Florida Entomologist. 168-174.

De Barro, P., Driver, F., 1997. Use of RAPD PCR to distinguish the B biotype from other biotypes of Bemisia tabaci (Gennadius)(Hemiptera: Aleyrodidae). Australian Journal of Entomology. 36, 149-152.

Elbert, A., Nauen, R., 2000. Resistance of Bemisia tabaci (Homoptera: Aleyrodidae) to insecticides in southern Spain with special reference to neonicotinoids. Pest Management ence. 56.

Erdogan, C., et al., 2008. Insecticide resistance and biotype status of populations of the tobacco whitefly Bemisia tabaci (Hemiptera: Aleyrodidae) from Turkey. Crop Protection. 27, 600-605.

Freitas, J. A., et al., 2002. Inheritance of foliar zingiberene contents and their relationship to trichome densities and whitefly resistance in tomatoes. Euphytica. 127, 275-287.

$\mathrm{Hu}$, J., et al., 2011. An extensive field survey combined with a phylogenetic analysis reveals rapid and widespread invasion of two alien whiteflies in China. PloS one. 6.

Ingham, V. A., et al., 2020. A sensory appendage protein protects malaria vectors from pyrethroids. Nature. 577, 1-5.

Kim, D., et al., 2019. Graph-based genome alignment and genotyping with HISAT2 and HISAT-genotype. Nature biotechnology. 37, 907-915.

Langfelder, P., Horvath, S., 2008. WGCNA: an R package for weighted correlation network analysis. BMC bioinformatics. 9, 559.

Leal, Walter, S., 2013. Odorant Reception in Insects: Roles of Receptors, Binding Proteins, and Degrading Enzymes. Annual Review of Entomology. 58, 373-391.

Liao, Y., et al., 2014. featureCounts: an efficient general purpose program for assigning sequence reads to genomic features. Bioinformatics. 30, 923-930.

Ling, Z., et al., 2015. Insect herbivory elicits genome $\square$ wide alternative splicing responses in $\mathrm{N}$ icotiana attenuata. The Plant Journal. 84, 228-243.

Muigai, S., et al., 2002. Mechanisms of resistance inLycopersicon germplasm to the whiteflyBemisia argentifolii. Phytoparasitica. 30, 347.

Pelosi, P., et al., 2017. Beyond chemoreception: Diverse tasks of soluble olfactory proteins in insects. Biological Reviews of the Cambridge Philosophical Society. 93. 
Robinson, M. D., et al., 2010. edgeR: a Bioconductor package for differential expression analysis of digital gene expression data. Bioinformatics. 26, 139-140.

Shen, S., et al., 2014. rMATS: robust and flexible detection of differential alternative splicing from replicate RNA-Seq data. Proceedings of the National Academy of Sciences. 111, E5593-E5601.

Song, S., et al., 2012. Enantioselective iridium $\square$ catalyzed hydrogenation of $\beta, \gamma \square$ unsaturated carboxylic acids: An efficient approach to chiral $4 \square$ alkyl $\square 4 \square$ aryl butanoic acids. Angewandte Chemie International Edition. 51, 2708-2711.

Su, Q., et al., 2016. Tomato yellow leaf curl virus differentially influences plant defence responses to a vector and a non $\square$ vector herbivore. Plant, cell \& environment. 39, 597-607.

Suh, E., et al., 2014. Peripheral olfactory signaling in insects. Curr Opin Insect Sci. 6, 86-92.

Sultan, M., et al., 2008. A global view of gene activity and alternative splicing by deep sequencing of the human transcriptome. Science. 321, 956-960.

Wang, E. T., et al., 2008. Alternative isoform regulation in human tissue transcriptomes. Nature. $456,470-476$.

Wei, J., et al., 2017. Vector development and vitellogenin determine the transovarial transmission of begomoviruses. Proceedings of the National Academy of Sciences. 114, 6746-6751.

Xie, W., et al., 2017. Genome sequencing of the sweetpotato whitefly Bemisia tabaci MED/Q. GigaScience. 6, gix 018 . 
Figure legends

Fig.1: GC-MS analysis of the volatile profiles of wild tomato Solanum habrochaites LA2175, LA2329, and common tomato Xianke 8. 1. R-curcumene, 2. 7-epizingiberene, 3 . p-cymene, 4. $\beta$-phellandrene, 5. elemene, 6. $\alpha$-copaene, 7. unknow, 8. $\beta$-caryophyllene, 9. $\alpha$-humulene.

Fig.2: Analysis of the RNA-Seq data of whiteflies treated by common tomato and wild tomato. (A) Principal component analysis (PCA) plot showing clustering of RNA-Seq of whiteflies. (B) Bar graph showing the number of upregulated (upper bars) and downregulated (lower bars) genes in whitefly male and female adults. (C) Venn diagram showing the number of induced differently expressed genes unique to or common within the four conditions. (D) Venn diagram showing the number of suppressed differently expressed genes unique to or common within the four conditions. WF6: wild tomato (Solanum habrochaites) 6 hours treated female adults; WM6: wild tomato 6 hours treated male adults; WF24: wild tomato 24 hours treated female adults; WM24: wild tomato 24 hours treated male adults. 
bioRxiv preprint doi: https://doi.org/10.1101/2021.02.26.432993; this version posted March 24, 2021. The copyright holder for this preprint (which was not certified by peer review) is the author/funder. All rights reserved. No reuse allowed without permission.

Fig.1
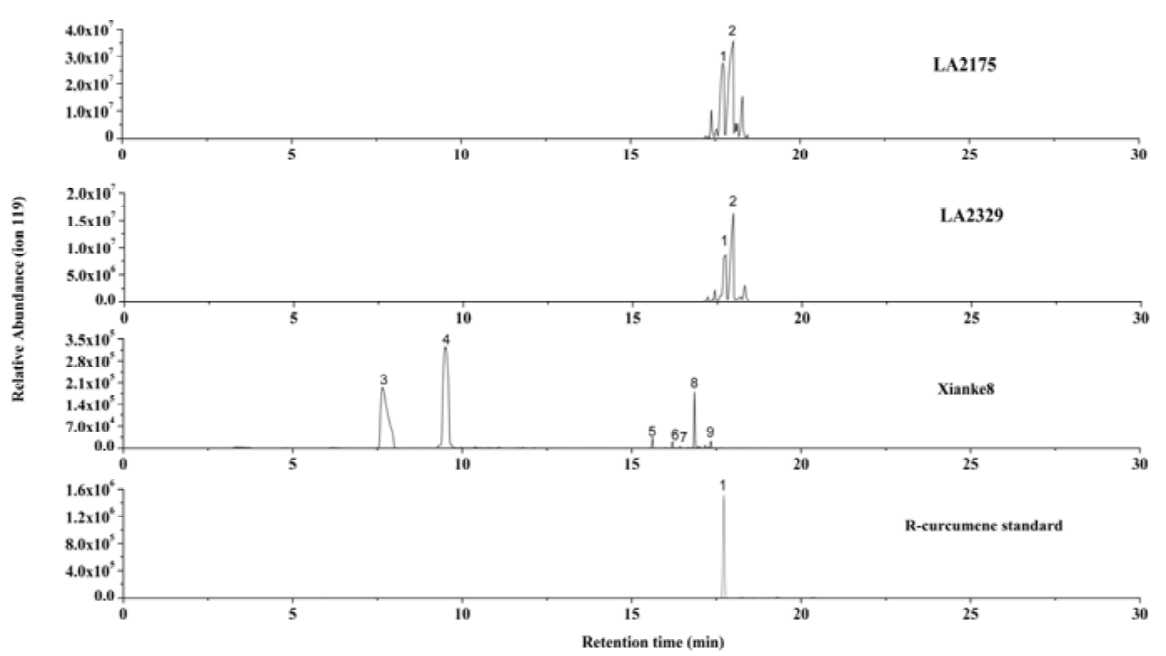
Fig.2

A

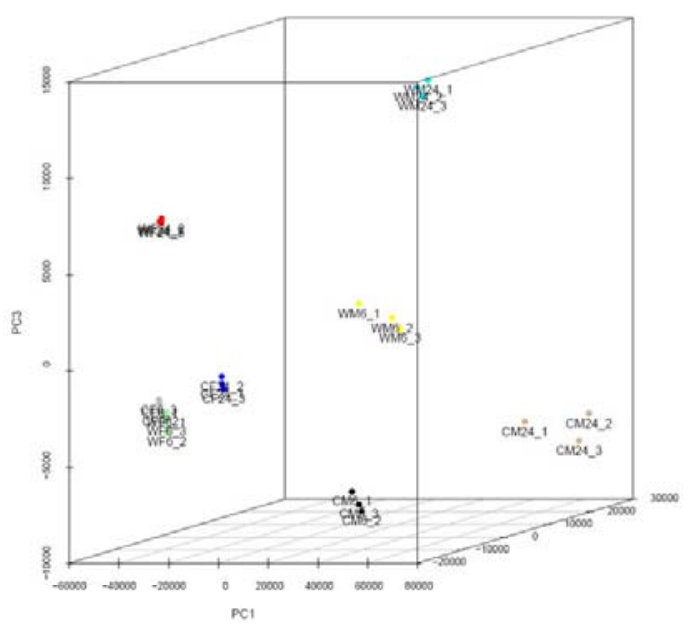

C

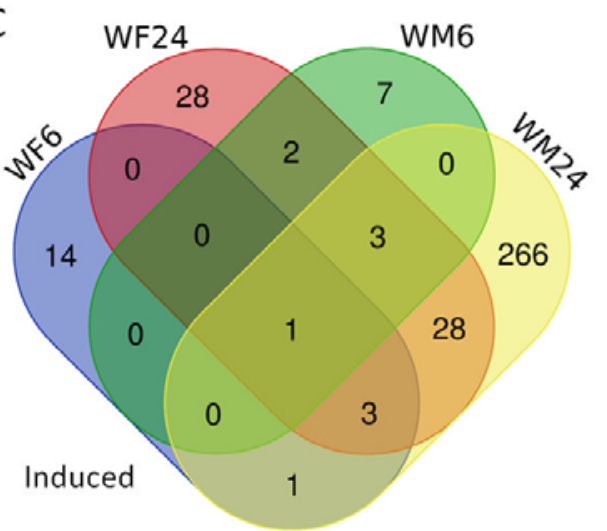

B

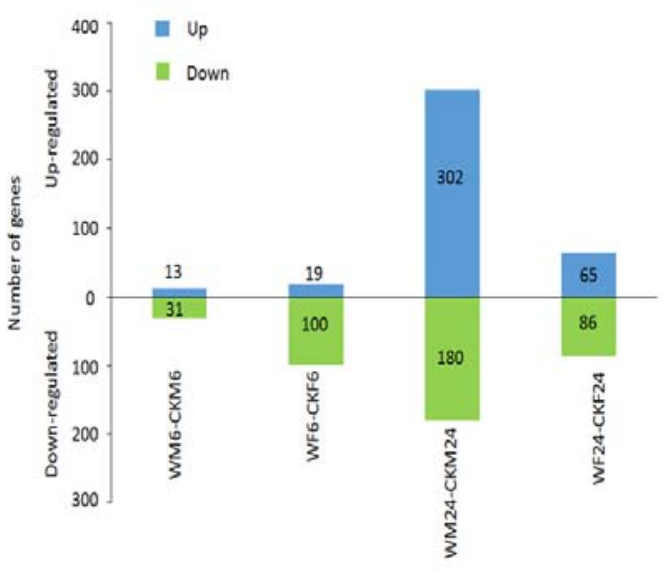

D

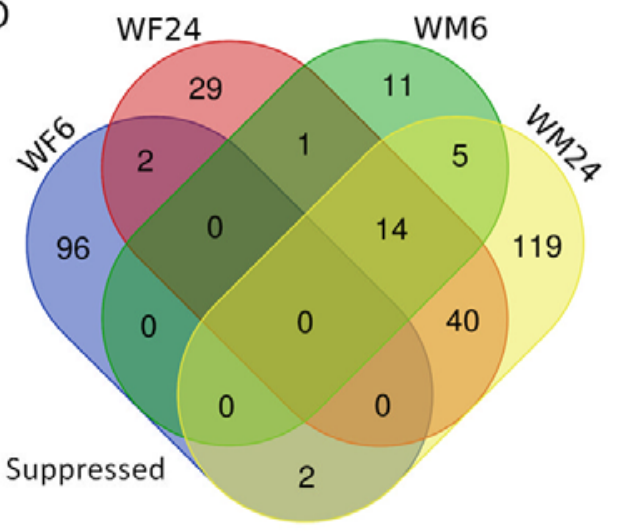


Table legends

Table 1. Summary of the RNA-Seq Data. CKF6_1-3, whitefly females grown on common tomato for $6 \mathrm{~h}$ replicates 1-3. CKM6_1-3, whitefly males grown on common tomato for $6 \mathrm{~h}$ replicates 1-3. CKF24_1-3, whitefly females grown on common tomato for $24 \mathrm{~h}$ replicates $1-3$. CKM24_1-3, whitefly males grown on common tomato for $24 \mathrm{~h}$ replicates $1-3$. WF6_1-3, whitefly females grown on wild tomato LA2175 for $6 \mathrm{~h}$ replicates $1-3$. WM6_1-3, whitefly females grown on wild tomato LA2175 for 6 h replicates $1-3$. WF24_1-3, whitefly females grown on wild tomato LA2175 for 24 h replicates $1-3$. WM24_1-3, whitefly males grown on wild tomato LA2175 for $24 \mathrm{~h}$ replicates $1-3$.

Table 2. Genome-wide effects of Solanum habrochaites stress on alternative splicing of whitefly. WF6: wild tomato (Solanum habrochaites) 6-h-treated female adults; WM6: wild tomato 6-h-treated male adults; WF24: wild tomato 24-h-treated female adults; WM24: wild tomato 24-h-treated male adults. CKF6: common tomato 6-h-treated female adults; CKM6: common tomato 6-h-treated male adults; CKF24: common tomato 24-h-treated female adults; CKM24: common tomato 24-h-treated male adults. 
bioRxiv preprint doi: https://doi.org/10.1101/2021.02.26.432993; this version posted March 24, 2021. The copyright holder for this preprint (which was not certified by peer review) is the author/funder. All rights reserved. No reuse allowed without permission.

Table 1

\begin{tabular}{|c|c|c|c|}
\hline & No. of paired-end reads & No. of mapped paired-end reads & Proportion of mapped paired-end reads \\
\hline CKF6_1 & 22729871 & 19170373 & $84.34 \%$ \\
\hline CKF6_2 & 24263436 & 20446798 & $84.27 \%$ \\
\hline CKF6_3 & 24587112 & 20852330 & $84.81 \%$ \\
\hline CKM6_1 & 22886414 & 18199276 & $79.52 \%$ \\
\hline CKM6_2 & 22949620 & 18146265 & $79.07 \%$ \\
\hline CKM6_3 & 22716792 & 18100740 & $79.68 \%$ \\
\hline CKF24_1 & 22159172 & 18759955 & $84.66 \%$ \\
\hline CKF24_2 & 22190660 & 18620183 & $83.91 \%$ \\
\hline CKF24_3 & 23055250 & 19278800 & $83.62 \%$ \\
\hline CKM24_1 & 22912092 & 18384663 & $80.24 \%$ \\
\hline CKM24_2 & 22576192 & 17744887 & $78.60 \%$ \\
\hline CKM24_3 & 22106168 & 17342289 & $78.45 \%$ \\
\hline WF6_1 & 21695473 & 17994225 & $82.94 \%$ \\
\hline WF6_2 & 25184704 & 21089671 & $83.74 \%$ \\
\hline WF6_3 & 24590350 & 20636222 & $83.92 \%$ \\
\hline WM6_1 & 23754024 & 19112488 & $80.46 \%$ \\
\hline WM6_2 & 24277496 & 19358875 & $79.74 \%$ \\
\hline WM6_3 & 22011538 & 17624639 & $80.07 \%$ \\
\hline WF24_1 & 22934225 & 19340432 & $84.33 \%$ \\
\hline WF24_2 & 22669251 & 18994565 & $83.79 \%$ \\
\hline WF24_3 & 24285715 & 20487429 & $84.36 \%$ \\
\hline WM24_1 & 23292401 & 19060172 & $81.83 \%$ \\
\hline WM24_2 & 21426439 & 17460405 & $81.49 \%$ \\
\hline WM24_3 & 24205186 & 19790160 & $81.76 \%$ \\
\hline
\end{tabular}


bioRxiv preprint doi: https://doi.org/10.1101/2021.02.26.432993; this version posted March 24, 2021. The copyright holder for this preprint (which was not certified by peer review) is the author/funder. All rights reserved. No reuse allowed without permission.

Table2

\begin{tabular}{|l|r|l|l|r|}
\hline AS Events Summary & $\begin{array}{l}\text { WF6 vs } \\
\text { CKF6 }\end{array}$ & $\begin{array}{l}\text { WM6 vs } \\
\text { CKM6 }\end{array}$ & $\begin{array}{l}\text { WF24 vs } \\
\text { CKF24 }\end{array}$ & $\begin{array}{l}\text { WM24 vs } \\
\text { CKM24 }\end{array}$ \\
\hline SE & 7 & 13 & 11 & 14 \\
\hline MXE & 0 & 1 & 2 & 5 \\
\hline RI & 0 & 0 & 0 & 0 \\
\hline A5SS & 0 & 0 & 0 & 0 \\
\hline A3SS & 0 & 0 & 0 & 0 \\
\hline
\end{tabular}

